\title{
PLANE AFFINE GEOMETRY AND ANOSOV FLOWS
}

\author{
BY THIERRY BARBOT
}

\begin{abstract}
We study Anosov flows on closed 3-manifolds. We define the notion of Anosov flows with the topological contact property (abreviation TCP Anosov flows): typical examples of TCP Anosov flows are contact Anosov flows, i.e. flows preserving a contact 1-form. We show that TCP Anosov flows are $\mathbf{R}$-covered. The main tool is the study of the leaf spaces of lifted strong stable foliations: we exhibit on these leaf spaces a structure of (generalized) affine plane, in the sense of incidence geometry.
\end{abstract}

() 2001 Éditions scientifiques et médicales Elsevier SAS

RÉSUMÉ. - Nous étudions les flots d'Anosov sur les variétés fermées de dimension 3. Nous introduisons la notion de flots d'Anosov topologiquement de contact : les exemples typiques de tels flots sont les flots d'Anosov de contact, i.e. ceux qui préservent une 1-forme de contact. Nous montrons que les flots d'Anosov topologiquement de contact sont produits. L'outil principal est l'étude de l'espace des feuilles de chaque feuilletage fort relevé dans le revêtement universel : nous exhibons sur ces espaces des feuilles une structure de plan affine (généralisé) au sens de la géométrie d'incidence.

○ 2001 Éditions scientifiques et médicales Elsevier SAS

\section{Introduction}

Let $M$ be a closed manifold equipped with an Anosov flow $\Phi^{t}$, i.e. a flow generated by a nonsingular $C^{1}$ vector field $X$ such that the tangent bundle $T M$ of $M$ splits continuously as a Whitney sum $T M=\mathbf{R} X \oplus E^{s s} \oplus E^{u u}$ of vector subbundles where:

- $\mathbf{R} X$ is the tangent line bundle defined by the flow direction $X$,

- $E^{s s}$ (respectively $E^{u u}$ ) is preserved and exponentially contracted (respectively expanded) by the differential of the flow.

$E^{s s}$ and $E^{u u}$ are called the stable and unstable strong directions. In general, they are only Hölder continuous (see [21]). They are uniquely integrable and define two foliations, the socalled strong stable and unstable foliations, and denoted by $\mathcal{F}^{s s}$ and $\mathcal{F}^{u u}$. When we add the flow direction $\mathbf{R} X$ to the strong directions, we obtain two plane fields which are uniquely integrable: they define two foliations $\mathcal{F}^{s}$ and $\mathcal{F}^{u}$, transverse one to the other, and both tangent to the flow. They are called the weak stable and unstable foliations. A very interesting property of Anosov flows is the structural stability: if $Y$ is a vector field sufficiently near to $X$ in the $C^{1}$-topology, then it generates an Anosov flow $\Psi^{t}$ which is topologically equivalent to $\Phi^{t}$, i.e. there exists a homeomorphism from the ambient manifold to itself, mapping the orbits of $\Phi^{t}$ on orbits of $\Psi^{t}$ (but the parametrisations are not necessarily preserved).

Typical examples of Anosov flow are the suspensions of Anosov diffeomorphisms: a diffeomorphism $f: M \rightarrow M$ is Anosov if the tangent bundle of $M$ splits continuously as the sum of two subbundles, one which is exponentially contracted and the other exponentially expanded. The suspension of $f$ is the flow induced by the vector field $\frac{\partial}{\partial t}$ on the quotient of 
$M \times \mathbf{R}$ by the relation identifying $(x, t)$ with $(f(x), t-1)$. The characteristic property of Anosov flows topologically equivalent to suspensions is the existence of a cross-section. The only known examples of Anosov diffeomorphisms are hyperbolic automorphisms of infranilmanifolds ([11]), and it is conjectured that this is the only possibility. According to [25] and [11], if an Anosov diffeomorphism is such that its stable or unstable direction is of dimension one, then it is topologically conjugate to a linear hyperbolic automorphism of a torus. Moreover, A. Verjovsky ([34]) conjectured that every Anosov flow for which one of the strong direction is of dimension one, and on a closed manifold of dimension strictly higher than three, is topologically equivalent to the suspension of an Anosov diffeomorphism. S. Simić proved this conjecture with the additional hypothesis that the one-dimensional direction is $\alpha$-Hölder for any $\alpha<1$ ([31]).

Another important family of Anosov flows are the geodesic flows on unitary tangent bundles of closed manifolds equipped with a riemannian metric of negative curvature (cf. [1]). These flows are not suspensions, but there is no contradiction with the discussion above since such a flow has a strong direction of dimension one if and only if the riemannian manifold is of dimension two, i.e. only when the ambient manifold is of dimension three. From this observation we see that the 3-dimensional case is quite particular. Actually, there is a great variety of Anosov flows in dimension three ([12,19,5,4]). D. Fried gave a description of Anosov flows on 3-manifolds which are transitive, i.e. which admits a dense orbit, as a kind of twisted suspension of certain pseudo-Anosov maps on surfaces ([13]). This description, quite general, has the same advantage and the same limit than the description of 3 -manifolds by surgeries along links of $\mathbf{S}^{3}$. It does not solve the problem of deciding, for example, what are the closed 3-manifolds admitting Anosov flows. Some facts are known in this direction: the universal covering of such a manifold is homeomorphic to $\mathbf{R}^{3}$ ([26]), its fundamental group has exponential growth ([29]). We gave partial results to this problem in the context of graphmanifolds ([3]). A particular feature in dimension 3 is the following: if $X$ is sufficiently smooth (i.e. of class $C^{2}$ ), then $\mathcal{F}^{s}$ and $\mathcal{F}^{u}$ are more regular than expected: they are of class $C^{1}$; more precisely, their tangent bundles $\mathbf{R} X \oplus E^{s s}$ and $\mathbf{R} \oplus E^{u u}$ are of class $C^{1}$ ([22]).

In dimension 3, we distinguish the special class of $\mathbf{R}$-covered ones. An Anosov flow is $\mathbf{R}$-covered if the lifting of one of the weak stable foliations in the universal covering of $M$ is a foliation by planes homeomorphic to the product foliation of $\mathbf{R}^{3}$ by horizontal planes $\mathbf{R}^{2} \times\{*\}$ (if one weak foliation lifts as a product foliation, the same is true for the other weak foliation [2, 8]). Not all Anosov flows are $\mathbf{R}$-covered (see [4]), but this property appears naturally when studying Anosov flows. For example, the equivalent property for Anosov diffeomorphisms is a key step in the proof of Frank's theorem, asserting that Anosov diffeomorphisms of codimension one are linear. It is also a crucial step in [28] and [14]. The classical examples (i.e. geodesic flows and suspensions) are $\mathbf{R}$-covered, and S. Fenley proved that the class of $\mathbf{R}$-covered Anosov flows is stable under Dehn-Goodman surgeries ([18]) satisfying a positivity condition, providing by the way a huge family of examples.

In [2], we investigated some properties of $\mathbf{R}$-covered Anosov flows not topologically equivalent to suspensions (see also [8] for similar and independent results). The main point is that these flows are characterized up to topological equivalence by some $C^{1}$-action of the fundamental group of the ambient manifold on the real line, the action commuting with a continuous homeomorphism without fixed point. This description of Anosov flows by an action on the line appears in [33]. We will recall in this paper the construction of this action. We will add the non-previously known fact (at least, to us) that the commuting homeomorphism is Hölder continuous.

In this paper, we study Anosov flows on closed 3-manifolds satisfying what we call the topological contact property (abbreviation TCP Anosov flows). This property means that there is no small loop, union of two small pieces of strong stable leaves and of two pieces of strong 
unstable leaves (for a precise definition, see Definition 3.5). This hypothesis is restrictive. For example, as a corollary of our results, the Bonatti-Langevin example ([5]), and its generalizations ([4]), are not TCP (because they are not $\mathbf{R}$-covered, see below), and thus, according to [4], it follows that there are 3-manifolds admitting Anosov flows, but no TCP Anosov flow. But the TCP hypothesis is valid in many interesting situations; for example, in the case of geodesic flows, and more generally in the case of contact Anosov flows, i.e. Anosov flows such that the sum $E^{s s} \oplus E^{u u}$ is a contact plane of class $C^{1}$ (cf. Lemma 3.7). P. Foulon proved that all the Anosov flows on graphmanifolds constructed by M. Handel and W. Thurston in [19] are contact. It is very presumable that the work of P. Foulon ([10], unfortunately a written version is not yet available) can be extended to a more general context. More precisely, the Dehn-Goodman surgery should be extended, in certain situations, to the context of contact Anosov flows. However, this optimistic point of view has to be tempered by the observation that Dehn-Goodman surgeries, applied to geodesic flows, can lead to suspensions of Anosov diffeomorphisms, and suspensions do not have the topological contact property (Proposition 5.1). It would be very interesting to decide whether the topological contact property is an open property amongst Anosov flows or not. More generally, we can wonder if the topological contact property is preserved by topological equivalence: it would provide a positive answer to the openness question, since Anosov flows are structurally stable. Proposition 5.1 is a first step in this direction.

A nice feature of TCP Anosov flows is the following (see Corollary 3.15):

\section{THEOREM A. - Any Anosov flow admitting the topological contact property is $\mathbf{R}$-covered.}

This theorem, in the case of contact Anosov flows, has been announced by V.V. Solodov in [32]. The proof of Solodov has never been published. The only known examples $\mathbf{R}$-covered Anosov flows which are not TCP are the flows topologically equivalent to suspensions (see Proposition 5.1).

As suggested by the title, there is a link between TCP Anosov flows and affine geometry. This link is very natural. A paradigm of this phenomenon is the case of geodesic flows of surfaces with constant negative curvature: the strong stable foliations associated to these flows are transversely affine. Let us be more precise: the geodesic flow in the constant negative curvature case can be described as the action by right translations of positive diagonal matrices on left compact quotients $M$ of $S L(2, \mathbf{R})$ by a discrete subgroup $\bar{\Gamma}$. The strong stable leaves are the orbits of the action by right translations of the group of upper unipotent matrices; the orbit space of this action is homeomorphic to the plane $\mathbf{R}^{2}$ minus the origin, and the natural action of $\bar{\Gamma}$ is conjugate to the usual linear action of $\bar{\Gamma}$ on $\mathbf{R}^{2} \backslash\{0\}$.

In order to see how this result can be generalized, we have to remind the notion of (axiomatic) affine plane. On the subject, we used the recent quite complete reference books [7,30]). An affine plane in the general meaning is a structure consisting of a set of points, with a collection of distinguished subsets called lines, satisfying the following three axioms:

- any two points lie on a unique line;

- if $l$ is a line and $p$ a point, then there is a unique line containing $p$ and parallel to (i.e. either equal to or disjoint from) $l$;

- there exist three noncollinear points.

The construction of affine planes from any division ring $K$ is well-known, especially in the case $K=\mathbf{R}$. We need not recall this construction here. Of course, there are many examples of affine planes which are not associated to a division ring. We have the following beautiful and fundamental theorem:

THEOREM. - An affine plane is associated to a division ring if and only if it satisfies Desargues' Theorem. It is associated to a field if and only if it satisfies Pappus' Theorem. 
Actually, we will consider only topological affine planes; i.e. we equip the set of points and the set of lines with a topology such that:

- the application mapping two different points on the line containing both is continuous,

- the application mapping two non parallel lines to their intersection point is continuous,

- the set of pairs of parallel lines is closed.

More precisely, we will actually assume that the topological affine plane is homeomorphic to $\mathbf{R}^{2}$, and that every line is closed in the plane and homeomorphic to $\mathbf{R}$ : these planes are the so-called affine $\mathbf{R}^{2}$-planes (see Chapter 3 of [30]).

The notion of affine $\mathbf{R}^{2}$-plane is not yet exactly the good notion associated to Anosov flows. The best picture to have in mind is as follows: remove a point $a$ from an affine $\mathbf{R}^{2}$-plane $A$, and take the universal covering of the remaining part. We obtain then a topological space $A^{*}$ with a collection of subsets which are the liftings of the lines in $A$, that we call generalized lines. There are two kinds of generalized lines: some of them project to lines in $A$ which do not contain the point $a$ : we call them complete lines. The others project in $A$ as half-lines, connected components of a line in $A$ minus $a$ : we call them rays. We say that two complete lines are parallel if and only if they intersect the same rays. Finally, for any complete line $l$, we call fundamental region associated to $l$ the union of the rays which intersect $l$. All the objects we defined satisfy the following axioms:

1. $A^{*}$ is homeomorphic to $\mathbf{R}^{2}$;

2. The rays are the leaves of a product foliation of $A^{*}$;

3 . any two points belonging to the same fundamental region lie on a unique generalised line;

4. if $p$ is point in $A^{*}$, and $l$ a complete line intersecting the ray through $p$, there is a unique line containing $p$ and parallel to $l$;

5. if two complete lines intersect the same ray, either their intersection is non-empty, either they are parallel;

6. there is a topology on the set $G$ of generalised lines such that the incidence maps $A^{*} \times A^{*} \rightarrow G$ and $G \times G \rightarrow A^{*}$, where they are defined, are continuous.

A topological space equipped with a collection of rays and complete lines satisfying the 6 axioms above - with the conventions that a fundamental region is the union of rays meeting a given complete line, and that two complete lines are called parallel if they define the same fundamental region - is called a lifted affine $\widetilde{\mathbf{R}}^{2}$-plane. A homeomorphism between two lifted affine $\widetilde{\mathbf{R}}^{2}$-planes mapping rays on rays and complete lines on complete lines is called a collineation. (Theorem 32.9 of [30], in the case of affine 2-planes, suggests that the

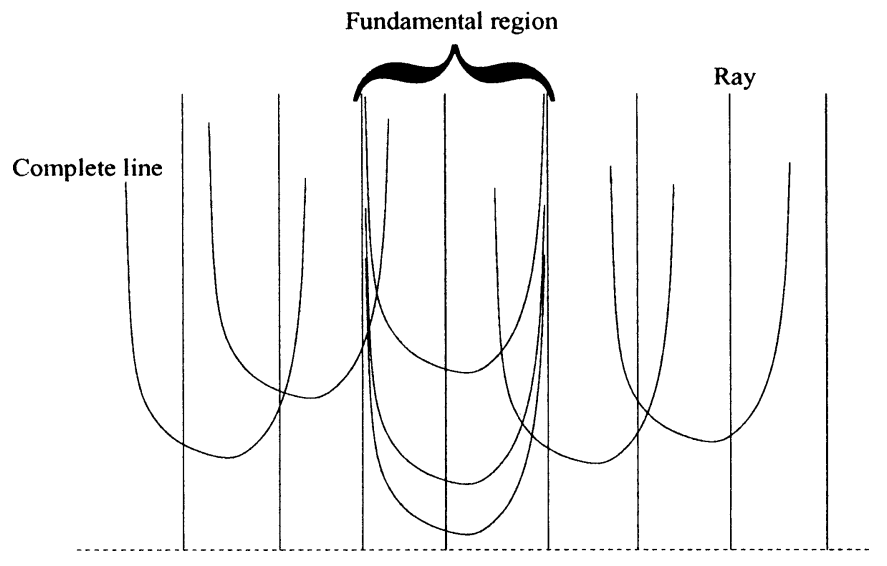

Fig. 1. Picture of a lifted affine $\widetilde{\mathbf{R}}^{2}$-plane. 
homeomorphism's assumption is a corollary of the preservation of the generalised lines. The details were not checked.)

Remark 1.1. - Let $A$ be an affine $\mathbf{R}^{2}$-plane, and select a line $l$ in $A$. Call rays the lines parallel to $l$, and complete lines the lines which are not parallel to $l$. Then, all these objects satisfy the six axioms of lifted affine $\widetilde{\mathbf{R}}^{2}$-planes, with the extra condition that there is only one fundamental region: the whole space itself. Conversely, any lifted affine $\widetilde{\mathbf{R}}^{2}$-plane with a unique fundamental region is actually an affine $\mathbf{R}^{2}$-plane with a distinguished direction of parallel lines. In this case, an affine collineation of the affine $\mathbf{R}^{2}$-plane is a collineation of the associated lifted affine $\widetilde{\mathbf{R}}^{2}$-planes if and only if it preserves the rays, i.e. it maps $l$ on a line parallel to $l$.

Consider now a TCP Anosov flow $\Phi^{t}$ on a closed 3-manifold $M$. Lift the strong foliations to foliations $\widetilde{\mathcal{F}}^{s s}$ and $\widetilde{\mathcal{F}}^{u u}$ in the universal covering $\widetilde{M}$ of $M$. Let $P^{s}$ be the leaf space of $\widetilde{\mathcal{F}}^{s s}$, i.e. the quotient of $\widetilde{M}$ by the equivalence relation identifying two points if they are on the same leaf of $\widetilde{\mathcal{F}}^{s s}$. It is not clear from this definition that the topology on this quotient set is Hausdorff, but it is true; actually we will show that $P^{s}$ is homeomorphic to $\mathbf{R}^{2}$ (Lemma 3.13). We denote by $p^{s}: \widetilde{M} \rightarrow P^{s}$ the quotient map. The fundamental group $\Gamma$ of $M$ acts naturally on $P^{s}$. The lifting of $\Phi^{t}$ to $\widetilde{M}$ preserves for any time $t$ the foliation $\widetilde{\mathcal{F}}^{s s}$. Therefore, it induces a flow $\varphi^{t}$ on $P^{s}$, which commutes with the $\Gamma$-action. Call rays the orbits of $\varphi^{t}$, and complete lines the projection by $p^{s}$ of leaves of $\widetilde{\mathcal{F}}^{u u}$. Then (see Theorem 3.17):

THEOREM B. - For any Anosov flow with the topological contact property, the leaf space $P^{s}$, with the collection of rays and complete lines defined as above is a lifted affine $\widetilde{\mathbf{R}}^{2}$-plane, whose collineation group contains $\Gamma$ and the flow $\varphi^{t}$.

Theorem A is precisely Axiom 2, its proof consists essentially in checking Axiom 1 and the uniqueness ingredient of Axiom 4 ; thus, one interest of Theorem B is that it provides an efficient way to remember the proof of Theorem A. In a forthcoming paper, we will see that the lifted affine $\widetilde{\mathbf{R}}^{2}$-plane $P^{s}$ is the universal covering of some punctured affine $\mathbf{R}^{2}$-plane if and only if the Anosov flow is a finite covering of an Anosov flow on the unitary tangent bundle of a surface. Therefore, contact Anosov flows (for example, geodesic flows) on unitary tangent bundles over surfaces provide examples of $\mathbf{R}^{2}$-planes. In general, these affine $\mathbf{R}^{2}$-planes are not isomorphic to the usual affine plane $\mathbf{R}^{2}$; we will see that it is the case if and only if the Anosov flow is topologically conjugate to the geodesic flow of a Riemannian surface with constant negative curvature, or to one of the "special reparametrizations" of it introduced in [15] (Proposition 6.2). Finally, we should mention other important facts that are discussed in Section 4:

- any TCP Anosov flow is topologically conjugate to the radial flow induced on the quotient of the flag variety associated to $P^{s}$ by the action of the fundamental group $\Gamma$,

- up to constant factors, there is a unique $\Gamma$-invariant Borel measure $\nu$ on the leaf space $P^{s}$ : the Margulis measure.

\section{R-covered Anosov flows}

We recall here the properties of $\mathbf{R}$-covered Anosov flows stated in [2]. Let $\left(M, \Phi^{t}\right)$ a $\mathbf{R}$-covered Anosov flow. We assume that $\Phi^{t}$ is not topologically equivalent to a suspension. Let $\widetilde{\Phi}^{t}$ be the lifted flow in the universal covering $\widetilde{M}$ of $M$. The orbit space $Q^{\Phi}$ of this lifted flow is diffeomorphic to $\mathbf{R}^{2}$ (Theorem 3.1 of [2], [8]). Let $\widetilde{\mathcal{F}}^{s}$ and $\widetilde{\mathcal{F}}^{u}$ be the liftings of the weak foliations in $\widetilde{M}$. Let $\mathcal{L}^{s}$ and $\mathcal{L}^{u}$ be the leaf spaces of these foliations. The $\mathbf{R}$-covered hypothesis means that $\mathcal{L}^{s}$ or $\mathcal{L}^{u}$ is a manifold homeomorphic to $\mathbf{R}$; actually if it is true for one of them, it is true for the other (Theorem 4.1 of [2]). 
The action of the fundamental group $\Gamma$ of $M$ on $\widetilde{M}$ induces natural actions on the various quotient spaces defined above.

There is a natural $\Gamma$-equivariant map $i: Q^{\Phi} \rightarrow \mathcal{L}^{s} \times \mathcal{L}^{u}$ : for any orbit $\tilde{\theta}$, the image $i(\tilde{\theta})$ is the pair of stable and unstable leaves containing $\tilde{\theta}$. The map $i$ is a homeomorphism onto its image, and, since we assumed that $\Phi^{t}$ is not topologically equivalent to a suspension, it is not surjective (Theorem 2.7 of [2]). Actually, the image of $i$ is the open set in $\mathcal{L}^{s} \times \mathcal{L}^{u}$ bounded by the graphs of two $\Gamma$-equivariant homeomorphisms $\alpha$ and $\beta$ from $\mathcal{L}^{s}$ onto $\mathcal{L}^{u}$. Let $\tau^{s}$ and $\tau^{u}$ be the compositions $\alpha^{-1} \circ \beta$ and $\beta \circ \alpha^{-1}$ : they are increasing homeomorphisms of $\mathcal{L}^{s}$ and $\mathcal{L}^{u}$. According to Theorem 4.6 of [2], there is a homeomorphism $I$ of $M$ onto itself, isotopic to the identity, realizing a non-trivial topological equivalence of $\Phi^{t}$ with its inverse (i.e. mapping an oriented orbit onto another orbit with the reversed orientation), and which lifts in $\widetilde{M}$ as a homeomorphism $\tilde{I}$ which induces on the quotient $Q^{\Phi} \subset \mathcal{L}^{s} \times \mathcal{L}^{u}$ the map $\left(l^{s}, l^{u}\right) \mapsto\left(\alpha^{-1}\left(l^{u}\right), \beta\left(l^{s}\right)\right)$.

\section{Proposition 2.1. - The maps $\tau^{s}$, $\alpha$ and $\beta$ are Hölder continuous.}

Remark 2.2. - Since R-covered Anosov flows are topologically transitive (Theorem 2.5 of [2]) and since we assume that the flow is not topologically equivalent to a suspension, every strong leaf is dense (Theorem 1.8 of [27]). It is easy to show from this property that the flow is topologically mixing (see, e.g., Chapter 18.3 of [20]).

Proof. - Consider the topological equivalence $I$ constructed above. According to Theorem 19.1.5 of [20], there is another Hölder continuous orbit equivalence $I^{\prime}$ arbitrarly $C^{0}$-close to $I$. Let $\widetilde{I}^{\prime}$ be the lifting of $I^{\prime} C^{0}$-close to $\widetilde{I}$. Let $\widetilde{\theta}$ be an orbit of $\widetilde{\Phi}^{t}$ preserved by an element $\gamma$ of $\Gamma$. Then, $\widetilde{I}(\widetilde{\theta})$ and $\widetilde{I}(\widetilde{\theta})$ are fixed points of $\gamma$ in $Q^{\Phi}$ which are very near. Hence, they are equal since $\gamma$-fixed points are discrete (actually, we use also the fact that the $\gamma$-fixed points are precisely the $\widetilde{I}$-iterates of $\widetilde{\theta}$ ). In other words, the induced actions of $I$ and $I^{\prime}$ in $Q^{\Phi}$ coincide on the projection of lifted periodic orbits. Since the flow is topologically transitive, periodic orbits are dense. It follows that the actions of $\widetilde{I}$ and $\widetilde{I}^{\prime}$ on $Q^{\Phi}$ are equal. Since $I^{\prime}$ is Hölder continuous, the proposition follows.

\section{TCP Anosov flows}

We no longer assume in this section that the Anosov flow $\Phi^{t}$ is $\mathbf{R}$-covered. Even in this case, the orbit space $Q^{\Phi}$ is Hausdorff, homeomorphic to $\mathbf{R}^{2}$. We assume that the strong foliations $\mathcal{F}^{s s}$ and $\mathcal{F}^{u u}$ are oriented: this is not a restriction up to finite coverings. We first recall a crucial result of S. Fenley ([9]) about non $\mathbf{R}$-covered Anosov flows. Since the lifted weak foliations are tangent to $\widetilde{\Phi}^{t}$, they induce two foliations $\mathcal{G}^{s}$ and $\mathcal{G}^{u}$ on $Q^{\Phi}$. They are foliations by closed lines; every leaf of one of these foliations intersects every leaf of the other foliation in at most one point. Moreover, they are $\Gamma$-invariant. Obviously, the flow is $\mathbf{R}$-covered if and only if the foliations $\mathcal{G}^{s}$ and $\mathcal{G}^{u}$ are individually conjugate to the product foliation of $\mathbf{R}^{2}$ by horizontal lines. We call open semileaf a connected component (in the leaf) of a leaf of $\mathcal{G}^{s}$ or $\mathcal{G}^{u}$ minus one point. A closed semileaf is the closure of an open semileaf. Obviously, we can speak of stable or unstable (semi)leaves.

DEFINITION 3.1. - A lozenge is an open subset of $Q^{\Phi}$ bounded by four closed semileaves $u_{1}$, $u_{2}, s_{1}, s_{2}$ such that:

- $s_{1}$ and $s_{2}$ are stable semileaves; $u_{1}$ and $u_{2}$ are unstable semileaves;

- $s_{i}$ and $u_{i}$ have a common point $x_{i}(i=1,2)$;

- a leaf of $\mathcal{G}^{u}$ (resp. of $\mathcal{G}^{s}$ ) meets $s_{1}$ (resp. $u_{1}$ ) if and only if it meets $s_{2}$ (resp. $u_{2}$ ). 
The points $x_{1}$ and $x_{2}$ are called the vertices of the lozenge; the semileaves $u_{1}, u_{2}, s_{1}$ and $s_{2}$ are called the sides of the lozenge. Let $\gamma$ be an element of the fundamental group $\Gamma$; the lozenge is $\gamma$-invariant if its vertices are preserved by $\gamma$.

Observe that the sides of a $\gamma$-invariant lozenge are preserved by $\gamma$.

Definition 3.2. - Two lozenges $L_{1}$ and $L_{2}$ are (un)stably adjacent if they are disjoint but such that their closures contain both the same (un)stable semileaf.

THEOREM 3.3 (S. Fenley [9]). - Let $\Phi^{t}$ be an Anosov flow on a closed 3-manifold M. Assume that $\Phi^{t}$ is not $\mathbf{R}$-covered. Let $u$ and $u^{\prime}$ be two leaves of $\mathcal{G}^{u}$ which are not separated by the quotient topology of $\mathcal{L}^{s}$. Then, there is an element $\gamma$ of $\Gamma$, and a finite sequence of distinct $\gamma$-invariant lozenges $L_{1}, \ldots, L_{k}$ such that:

- each $L_{i}$ is stably adjacent to the following $L_{i+1}$,

- $u$ contains a side of $L_{1}$,

- $u^{\prime}$ contains a side of $L_{k}$.

Select an auxiliary metric on $M$. The length metric on leaves defines continuous parametrizations of the oriented foliations $\mathcal{F}^{s s}$ and $\mathcal{F}^{u u}$; i.e. the leaves of these foliations are the orbits of flows that we denote respectively by $h_{s}^{t}$ and $h_{u}^{t}$. For any $\varepsilon>0$ and for any element $x$ of $M$, we denote by $\mathcal{F}_{\varepsilon}^{s s}(x)$ the $\varepsilon$-ball centered at $x$ in the leaf of $\mathcal{F}^{s s}$ through $x$ equipped with the induced length metric. We define similarly $\mathcal{F}_{\varepsilon}^{u u}(x), \mathcal{F}_{\varepsilon}^{s}(x)$ and $\mathcal{F}_{\varepsilon}^{u}(x)$.

Definition 3.4. - The Anosov flow has a $\varepsilon$-rectangle if there is a point $x$ in $M$ and four real numbers $t_{1}, t_{2}, t_{3}$ and $t_{4}$ different from zero and of absolute value less than $\varepsilon$ such that $h_{s}^{t_{1}} \circ h_{u}^{t_{2}}(x)=h_{u}^{t_{3}} \circ h_{s}^{t_{4}}(x)$.

Definition 3.5. - The Anosov flow has the topological contact property if there is a real positive number $\varepsilon_{0}$ such that the flow has no $\varepsilon_{0}$-rectangle.

There is a local product structure for the pair $\left(\mathcal{F}^{u}, \mathcal{F}^{s s}\right)$, i.e. there is a real positive number $\varepsilon$ such that, for any point $x$ in $M$ and any point $y$ in the $2 \varepsilon$-neighborhood of $x$, the local strong stable leaf $\mathcal{F}_{4 \varepsilon}^{s s}(y)$ meets the local leaf $\mathcal{F}_{4 \varepsilon}^{u}(x)$ at a unique point $\pi_{x}(y)$.

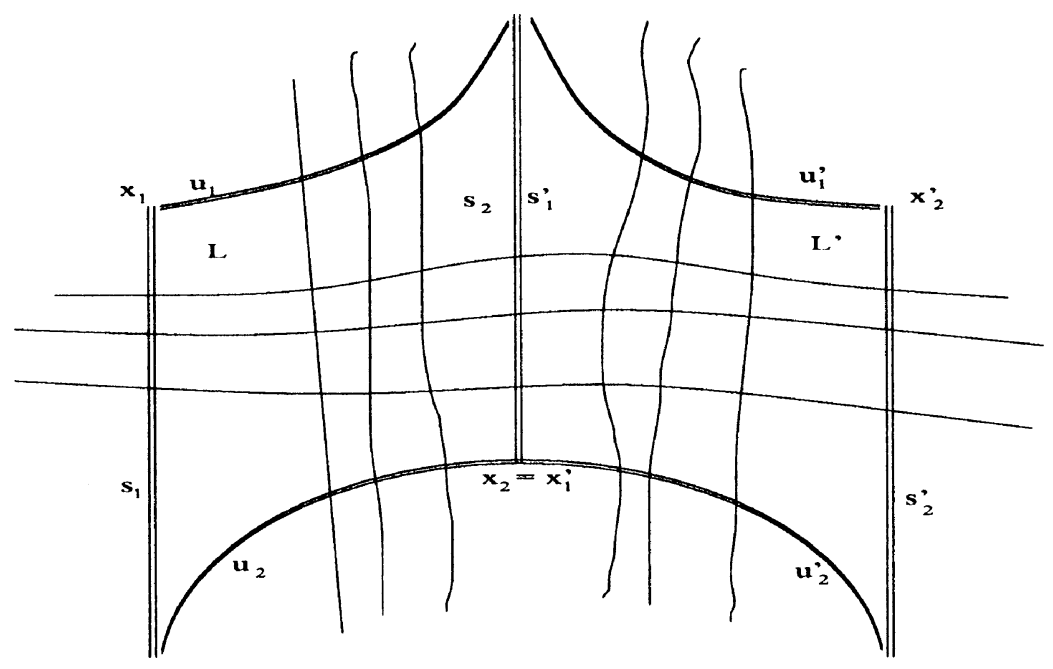

Fig. 2. Adjacent lozenges. 


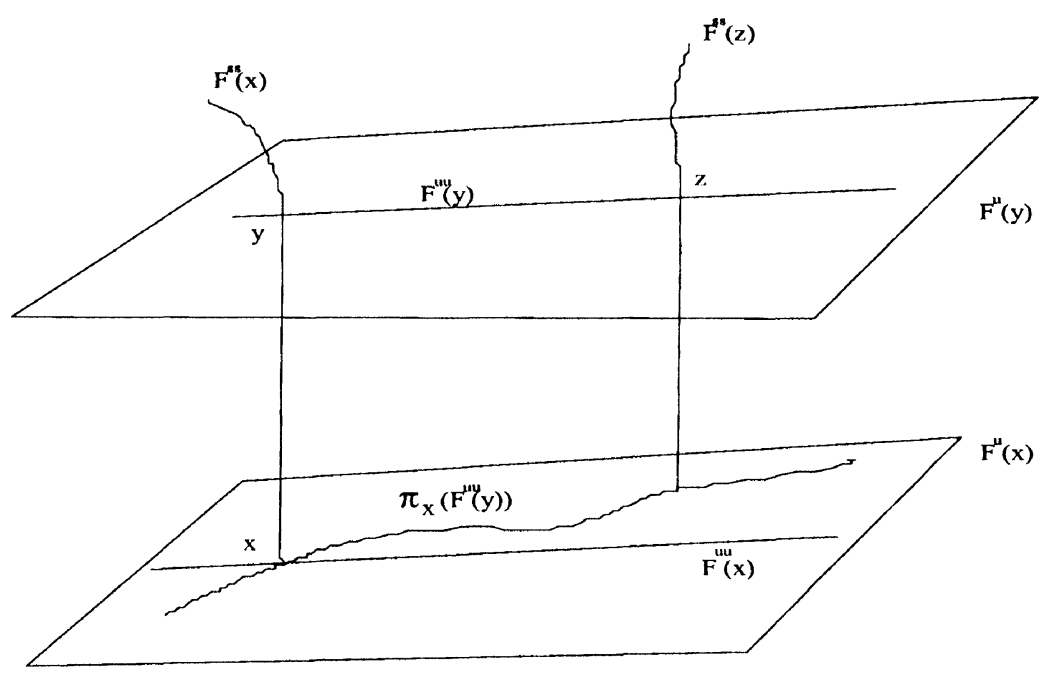

Fig. 3. The topological contact property.

The following lemma is obvious:

LEMA 3.6. - The Anosov flow has the topological contact property if and only if there is a real positive number $\varepsilon_{0}$ less than $\varepsilon$ such that, for any point $x$ in $M$, and for any point $y \neq x$ in the local leaf $\mathcal{F}_{\varepsilon_{0}}^{s s}(x)$, the projection $\pi_{x}\left(\mathcal{F}_{\varepsilon_{0}}^{u u}(y)\right)$ meets $\mathcal{F}_{\varepsilon_{0}}^{u u}(x)$ only at the point $x$, and nowhere else.

A contact Anosov flow is an Anosov flow such that the plane bundle $P=E^{s s} \oplus E^{u u}$ is a contact plane of class $C^{1}$. Then, $E^{s s}$ is the intersection between the $C^{1}$-bundles $E^{s s} \oplus E^{u u}$ and $E^{s s} \oplus \mathbf{R} X$ : it is of class $C^{1}$. It is worth to know the following theorem of $\mathrm{S}$. Hurder and A. Katok (Theorem 2.3 of [23]): for any smooth contact Anosov flow, the contact plane is actually smooth. Moreover, a smooth Anosov flow which is not topologically equivalent to a suspension is contact as soon as its strong bundles are both of class $C^{1}$.

\section{LEMA 3.7. - Contact Anosov flows have the topological contact property.}

Proof. - Let $\left(M, \Phi^{t}\right)$ be a contact Anosov flow. Since $E^{s s}$ is of class $C^{1}$, the maps $\pi_{x}$ are all of class $C^{1}$. Select a point $x$ in $M$, and consider a point $y$ in the local leaf $\mathcal{F}_{\varepsilon_{0}}^{s s}(x)\left(\varepsilon_{0}<\varepsilon\right)$ and different from $x$. The local strong unstable leaves contained in $\mathcal{F}^{u}(y)$ are curves tangent to $E^{u u}$, which is the intersection between the tangent bundle of $\mathcal{F}^{u}(y)$ and $P$. Hence, $\pi_{x}\left(\mathcal{F}_{\varepsilon}^{u u}(y)\right)$ is tangent at every point $\pi_{x}(z)$ to the intersection of the tangent bundle of $\mathcal{F}^{u}(y)$ and $d \pi_{x}\left(P_{z}\right)$. But that $P$ is a contact plane means precisely that, at least for sufficiently small $\varepsilon_{0}, d_{z} \pi_{x}\left(P_{z}\right)$ is different from $P_{\pi_{x}(z)}$. Therefore, $\pi_{x}\left(\mathcal{F}_{\varepsilon}^{u u}(y)\right)$ is everywhere tangent to a local vector field which is nowhere tangent to the strong stable leaves. The lemma follows.

From now, we assume that the Anosov flow is TCP, i.e. admits the topological contact property. Let $s, t$ be two real positive numbers less than $\varepsilon_{0}$; for any element $x$ of $M$ define $\tau_{x}(s, t)$ as the unique time $\tau$ less than $4 \varepsilon$ such that $\Phi^{-\tau} \pi_{x}\left(h_{u}^{s} \circ h_{s}^{t}\right)$ belongs to $\mathcal{F}^{u u}(x)$. By definition of TCP Anosov flows, when $s$ and $t$ are not zero, $\tau_{x}(s, t)$ is not zero. Hence, all the $\tau_{x}(s, t)$ have the same sign. Reversing the orientation of one of the strong foliations, we can assume that this sign is positive. 
Definition 3.8. - Let $F_{1}$ and $F_{2}$ be different leaves of $\tilde{\mathcal{F}}^{u}$. Let $\Omega^{s}\left(F_{1}, F_{2}\right)$ be the intersection between $F_{1}$ and the $\widetilde{\mathcal{F}}^{s s}$-saturation of $F_{2}$. We denote by

$$
h^{s}\left(F_{1}, F_{2}\right): \Omega^{s}\left(F_{1}, F_{2}\right) \mapsto \Omega^{s}\left(F_{2}, F_{1}\right)
$$

the holonomy map along strong stable leaves.

LEMA 3.9. - For any pair $\left(F_{1}, F_{2}\right), \Omega^{s}\left(F_{1}, F_{2}\right)$ is an open connected subset of $F_{1}$ which is $\widetilde{\Phi}^{t}$-invariant.

Proof. - The openness and $\widetilde{\Phi}^{t}$-invariance are obvious. Let $G_{1}$ and $G_{2}$ be the projections of $F_{1}$ and $F_{2}$ in $Q^{\Phi}$ : they are leaves of $\mathcal{G}^{u}$. Since $Q^{\Phi}$ is diffeomorphic to $\mathbf{R}^{2}$, the set of points of $G_{1}$ whose $\mathcal{G}^{s}$-leaves meet $G_{2}$ is connected. The connectedness of $\Omega^{s}\left(F_{1}, F_{2}\right)$ follows immediatly.

When $\Omega^{s}\left(F_{1}, F_{2}\right)$ is not empty, the sign of the time needed to reach $F_{2}$ along the flow $\widetilde{h}_{s}$ and starting from $F_{1}$ is constant on $\Omega^{s}\left(F_{1}, F_{2}\right)$. If this sign is positive we write $F_{1} \prec F_{2}$, and we write $F_{1} \succ F_{2}$ if it is negative.

DEFINITION 3.10. - A graph on $F$ is a continuous path in $F$ which meets every orbit of $\widetilde{\Phi}^{t}$ in at most one point.

Let $F$ be a leaf of $\widetilde{\mathcal{F}}^{u}$ and $\widetilde{x}$ a point of $F$. The flows $\widetilde{\Phi}^{t}$ and $\widetilde{h}_{u}^{s}$ provide a parametrization of $F$ by $\mathbf{R}^{2}$, the point of coordinates $(t, s)$ being $\widetilde{\Phi}^{s} \circ \widetilde{h}_{u}^{t}(\widetilde{x})$. We call special parametrizations this type of parametrization. When we identify $F$ with $\mathbf{R}^{2}$ in this way, it is obvious that a continuous path $c$ in $F$ is a graph if and only if it is the graph in the usual meaning of some function $f$ from an interval of $\mathbf{R}$ into $\mathbf{R}$. If $f$ is (strictly) increasing (respectively decreasing), we say that $c$ is increasing (respectively decreasing). Observe that the notion of increasing and decreasing graphs does not depend on the parametrisation of the flow, but just on the orientations of $\widetilde{\Phi}$ and $\mathcal{F}^{u u}$.

Remark 3.11. - A decreasing or increasing graph in $F_{2}$ is a path which intersects any $\widetilde{\Phi}^{t}$-orbit in at most one point, and which intersects any $\widetilde{\mathcal{F}}^{u u}$-leaf in at most one point.

LEMA 3.12. - Let $F_{1}$ and $F_{2}$ be two leaves of $\widetilde{\mathcal{F}}^{u}$. We select the orientations of the strong foliations such that $F_{1} \prec F_{2}$ and such that $\tau_{x}(s, t)$ is positive for any pair $(s, t)$ in $] 0, \varepsilon_{0}[\times] 0, \varepsilon_{0}[$. Then, for any strong stable leaf $f^{u u}$ in $F_{1}$, the image of $f^{u u} \cap \Omega^{s}\left(F_{1}, F_{2}\right)$ by $h^{s}\left(F_{1}, F_{2}\right)$, if not empty, is an increasing graph.

Proof. - Denote by $c$ the image of $f^{u u} \cap \Omega^{s}\left(F_{1}, F_{2}\right)$ by $h^{s}\left(F_{1}, F_{2}\right)$, and assume that it is not empty. Since $f^{u u}$ meets every leaf of $\widetilde{\mathcal{F}}^{s}$ in at most one point, $c$ is a graph. To be an increasing graph is a local property, i.e. it is enough to see that for any point $x$ in $f^{u u} \cap \Omega^{s}\left(F_{1}, F_{2}\right)$, the graph $c$ is increasing near $h^{s}\left(F_{1}, F_{2}\right)(x)$. Replacing $x$ by some $\widetilde{\Phi}^{T}(x)$, we can assume that, near $x$, the time needed to reach $F_{2}$ (and thus $c$ ) along $\widetilde{\mathcal{F}}^{s s}$ is less than $\varepsilon_{0}$. Then, the positivity of the $\tau_{x}(s, t)$ is precisely what we need in order to show that $c$ is increasing near $h^{s}\left(F_{1}, F_{2}\right)(x)$.

Let $P^{s}$ be the leaf space of $\widetilde{\mathcal{F}}^{s}$. Let $p^{s}: \widetilde{M} \rightarrow P^{s}$ denote the projection map.

Proposition 3.13. - $P^{s}$ equipped with the quotient topology is homeomorphic to $\mathbf{R}^{2}$.

Proof. - The main difficulty is to prove the Hausdorff separation property for $P^{s}$. We argue by contradiction, assuming the existence of two different leaves $h_{1}$ and $h_{2}$ of $\widetilde{\mathcal{F}}^{s s}$ such that any $\widetilde{\mathcal{F}}^{s s}$-saturated neighborhood of $h_{1}$ meets any $\widetilde{\mathcal{F}}^{s s}$-saturated neighborhood of $h_{2}$. It implies that the $\widetilde{\mathcal{F}}^{s}$-leaves containing $h_{1}$ and $h_{2}$ are not separated one from the other. According to Theorem 3.3, there is an element $\gamma$ of $\Gamma$ preserving these two leaves. Hence, there are two real 

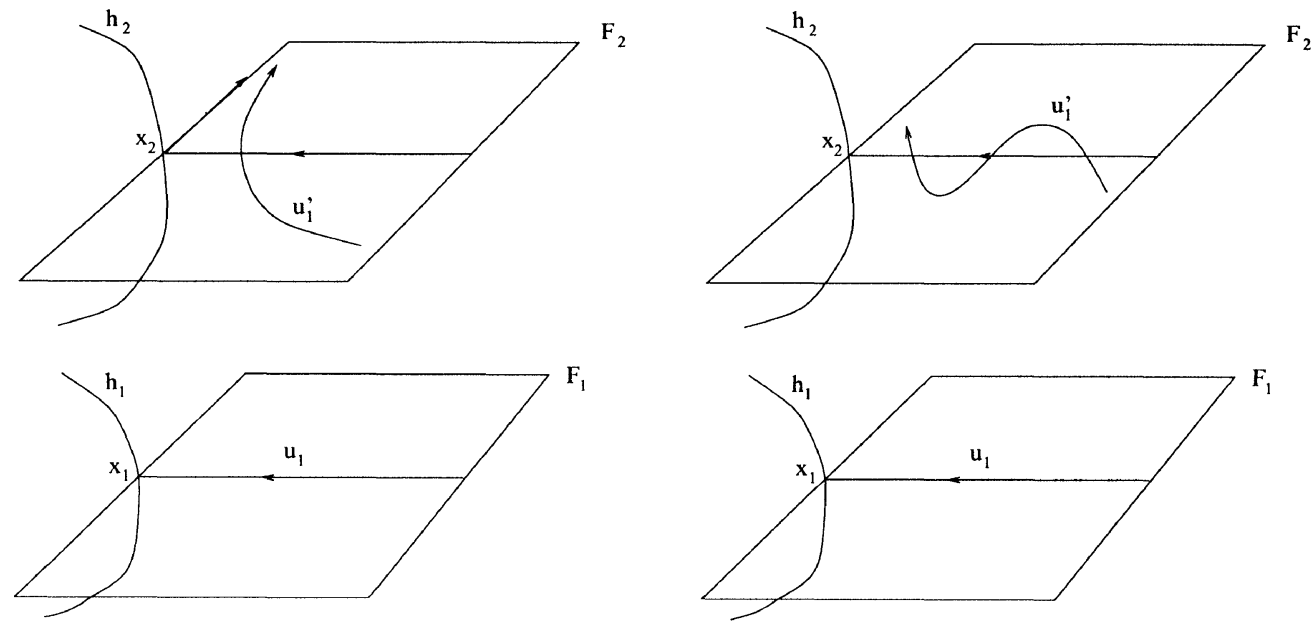

Case $T_{2}<T_{1}: u_{1}^{\prime}$ is asymptotic to the orbit through $\frac{x}{2}$

Case $T_{2}=T_{1}: u_{1}^{\prime}$ cannot be an increasing or decreasing graph

Fig. 4. Non-Hausdorff pair of strong stable leaves.

numbers $T_{1}$ and $T_{2}$ such that $\widetilde{\Phi}^{T_{i}} \gamma$ preserves $h_{i}(i=1,2)$. Moreover, $h_{i}$ contains a (unique) fixed point $x_{i}$ of $\widetilde{\Phi}^{T_{i}} \gamma(i=1,2)$. Let $F_{i}$ be the leaf of $\widetilde{\mathcal{F}}^{u}$ containing $x_{i}$. Since $h_{1}$ and $h_{2}$ are not separated, $x_{1}$ belongs to the closure of $\Omega^{s}\left(F_{1}, F_{2}\right)$; actually, since $h_{1}$ and $h_{2}$ are different, $x_{1}$ belongs to the boundary of $\Omega^{s}\left(F_{1}, F_{2}\right)$. Let $u_{1}$ be the intersection between $\Omega^{s}\left(F_{1}, F_{2}\right)$ and the leaf of $\widetilde{\mathcal{F}}^{u u}$ containing $x_{1}$, and let $u_{1}^{\prime}$ be the image of $u_{1}$ by $h^{s}\left(F_{1}, F_{2}\right)$. Since $F_{1}, F_{2}$ and $u_{1}$ are $\widetilde{\Phi}^{T_{1}} \gamma$-invariant, the same is true for $u_{1}^{\prime}$. Consider the special parametrization of $F_{2}$ by $\mathbf{R}^{2}$ for which $x_{2}$ is the origin. Then, the action on $F_{2}$ is of the form $(t, s) \mapsto\left(\kappa(t), s+T_{2}-T_{1}\right)$, where $\kappa$ is a contraction or dilatation of $\mathbf{R}$ admitting 0 as unique fixed point (it is conjugate to the action of $\widetilde{\Phi}^{T_{2}} \gamma$ on $\widetilde{\mathcal{F}} u u\left(x_{2}\right)$ ). If $T_{1}=T_{2}$, then every leaf of $\widetilde{\mathcal{F}}^{u u}$ in $F_{2}$ are $\widetilde{\Phi}^{T_{2}} \gamma$-invariant. But according to Lemma 3.12, such a leaf meets $u_{1}^{\prime}$ in at most one point. This is impossible since $u_{1}^{\prime}$ is $\gamma$-invariant (see Fig. 4). Therefore, $T_{2} \neq T_{1}$. It follows that $u_{1}^{\prime}$ is asymptotic to the $\widetilde{\Phi}^{t}$-orbit of $x_{2}$ (see Fig. 4).

Since $h_{1}$ and $h_{2}$ are not separated, there are elements $y_{n}$ of $\Omega^{s}\left(F_{1}, F_{2}\right)$ converging to $x_{1}$ and such that $y_{n}^{\prime}=h^{s}\left(F_{1}, F_{2}\right)\left(y_{n}\right)$ converge to $x_{2}$. Let $t_{n}$ be the unique real number such that $\widetilde{\Phi}^{t_{n}}\left(y_{n}\right)$ belongs to $u_{1}$ : the $t_{n}$ tend to 0 . On the other hand, $\widetilde{\Phi}^{t_{n}}\left(y_{n}^{\prime}\right)$ belongs to $u_{1}^{\prime}$. We obtain a contradiction since $u_{1}^{\prime}$ is asymptotic to the $\widetilde{\Phi}^{t}$-orbit of $x_{2}$ and the $y_{n}^{\prime}$ tend to $x_{2}$.

This contradiction shows that $P^{s}$ is Hausdorff. Now, every leaf of $\widetilde{\mathcal{F}}^{u}$ is homeomorphic to $\mathbf{R}^{2}$ and intersects every leaf of $\widetilde{\mathcal{F}}^{s s}$ in at most one point. Therefore, the restrictions of $p^{s}$ to leaves of $\widetilde{\mathcal{F}}^{u}$ are chards of some manifold structure on $P^{s}$. Moreover, if we select any parametrization $f^{t}$ of $\widetilde{\mathcal{F}}^{s s}$, for any leaf $F$ of $\widetilde{\mathcal{F}}^{u}$, the map $(x, t) \mapsto f^{t}(x)$ is a homeomorphism from $F \times \mathbf{R}$ onto the $\widetilde{\mathcal{F}}^{s s}$-saturation of $F$. It follows that $p^{s}$ is a locally trivial fibration by lines. Since $\widetilde{M}$ is homeomorphic to $\mathbf{R}^{3}, P^{s}$ is homeomorphic to $\mathbf{R}^{2}$.

The group $\Gamma$ acts naturally on $P^{s}$. Observe that this action is free since no leaf of $\mathcal{F}^{s s}$ is a circle. The flow $\widetilde{\Phi}$ defines a flow $\varphi^{t}$ on $P^{s}$. We call rays the orbits of $\varphi^{t}$, and complete lines the projections by $p^{s}$ of the leaves of $\widetilde{\mathcal{F}}^{u u}$. A generalized line is a ray or a complete line. The following lemma is an immediate corollary of Lemma 3.12 (see Remark 3.11):

LEMA 3.14. - The intersection between two generalized lines contains at most one point. 


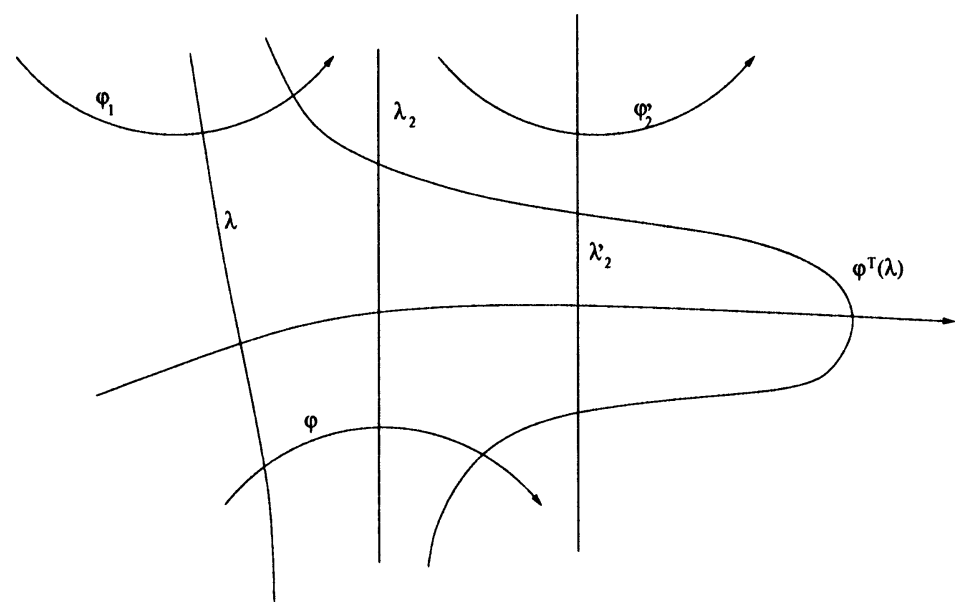

Fig. 5. Non-Hausdorff pair of orbits.

We can now deduce Theorem A:

\section{COROLlary 3.15. - TCP Anosov flows are $\mathbf{R}$-covered.}

Proof.-Assume that the TCP Anosov flow $\Phi^{t}$ is not $\mathbf{R}$-covered. Then, according to Theorem 3.3, $Q^{\Phi}$ contains two unstably adjacent lozenges $L$ and $L^{\prime}$. Let $u_{2}=u_{1}^{\prime}$ be the common side of $L$ and $L^{\prime}$; let $s_{1}$ and $s_{2}^{\prime}$ be the sides of $L$ and $L^{\prime}$ asymptotics to $u_{2}$, let $s_{2}, s_{1}^{\prime}$ be the other stable sides of $L, L^{\prime}$, and let be $u_{2}^{\prime}$ be the last unstable side of $L^{\prime}$. The sides $s_{2}, s_{1}^{\prime}$ are both contained in the same leaf $s$ of $\mathcal{G}^{s}$; more precisely, there is an element $\widetilde{\theta}$ of $s$ such that $s \backslash\{\widetilde{\theta}\}$ is the union of $s_{2}$ and $s_{1}^{\prime}$. Denote by $S, S_{1}, S_{2}^{\prime}, U_{2}$ and $U_{2}^{\prime}$ the preimage in $\widetilde{M}$ of $s, s_{1}, s_{2}^{\prime}, u_{2}$ and $u_{2}^{\prime}$ : the third ones are leaves of $\widetilde{\mathcal{F}}^{s}$, and the last ones are leaves of $\widetilde{\mathcal{F}}^{u}$. Project all these objects in $P^{s}$ : we obtain three rays $\varphi, \varphi_{1}$ and $\varphi_{2}^{\prime}$, and two complete lines $\lambda_{2}$ and $\lambda_{2}^{\prime}$ (see Fig. 5).

Moreover, near to $\lambda_{2}$, there is a complete line $\lambda$ which meets $\varphi_{1}$ and $\varphi$. Since the rays $\varphi_{1}$ and $\varphi_{2}^{\prime}$ are not separated, there is a ray near $\varphi_{1}$ which intersects $\lambda$ and $\lambda_{2}^{\prime}$. Hence, it follows from topological properties of lines in the plane that there is a time $T$ for which $\varphi^{T}(\lambda)$ intersects $\varphi_{1}, \lambda_{2}^{\prime}$ and $\varphi$. However, since $\varphi_{1}$ and $\varphi$ belong to the same connected component of $P^{s} \backslash \lambda_{2}^{\prime}$, this is possible only if $\varphi^{T}(\lambda)$ intersects $\lambda_{2}^{\prime}$ in at least two points. This is in contradiction with Lemma 3.14.

Our goal is to prove Theorem B, i.e. to check the six axioms of lifted affine $\widetilde{\mathbf{R}}^{2}$-planes for $P^{s}$. Observe that a fundamental region, which by definition is the union of rays which meets a given complete line, is in the context of TCP Anosov flows the projection of a leaf of $\widetilde{\mathcal{F}}^{u}$. Therefore, it is open. Moreover, two complete lines are parallel if and only if one is the image of the other by some $\varphi^{t}$.

LEMA 3.16. - Two points in $P^{s}$ belonging to the same fundamental region are contained in a unique generalized line.

Proof. - Let $s_{0}$ be an element $s_{0}$ of $P^{s}$, and $V$ any fundamental region containing $s_{0}$, i.e. the $\varphi^{t}$-saturation of some complete line $l_{0}$ containig $s_{0}$. Let $r_{0}$ be the ray through $s_{0}$. The line $l_{0}$ is the projection of some $\widetilde{\mathcal{F}}^{u u}$-leaf containing an element $x$ of the $\widetilde{\mathcal{F}}^{s s}$-leaf $s_{0}$, and, as observed previously, $V$ is the projection of the $\mathcal{F}^{u}$-leaf containing $x$. There is a special parametrization of $V$ by $\mathbf{R}^{2}$ such that $l_{0}$ is the horizontal line $\mathbf{R} \times\{0\}$, and $s_{0}$ the point $(0,0)$. Define the maps $f_{x}^{+}: \mathbf{R} \times \mathbf{R}_{+}^{*} \rightarrow P^{s}$ and $f_{x}^{-}: \mathbf{R} \times \mathbf{R}_{+}^{*} \rightarrow P^{s}$ by $f_{x}^{+}(s, t)=\widetilde{h}_{u}^{s} \circ \widetilde{h}_{s}^{t}(x)$ and 


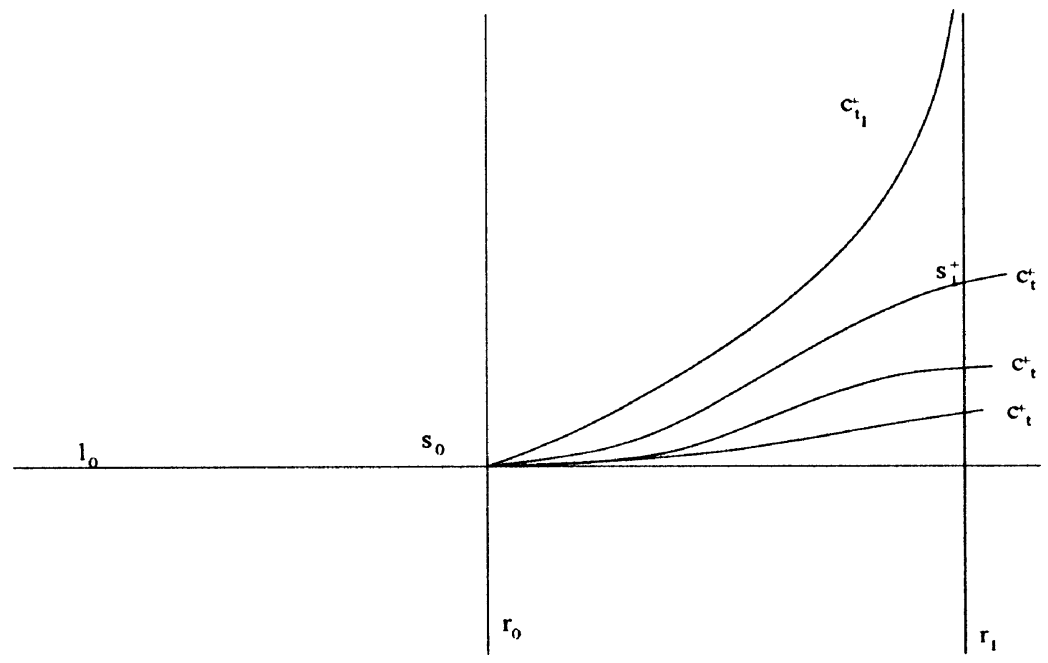

Fig. 6. Case 1.

$f_{x}^{-}(s, t)=\widetilde{h}_{u}^{s} \circ \widetilde{h}_{s}^{-t}(x)$ (where $\widetilde{h}_{u}^{s}$ and $\widetilde{h}_{s}^{t}(x)$ are parametrizations of $\widetilde{\mathcal{F}}^{s s}$ and $\widetilde{\mathcal{F}}^{u u}$ ). According to Lemma 3.14, these two maps are injective. Since they are continuous, and since $P^{s}$ is homeomorphic to $\mathbf{R}^{2}$, they are open. Denote by $U^{+}$and $U^{-}$their respective images. For any real number $t$, denote by $c_{t}^{ \pm}$the image of the map $s \mapsto f_{x}^{ \pm}(s, t)$.

Let $V^{+}$and $V^{-}$be the connected components of $V$ minus $r_{0}$ containing respectively $U^{+} \cap V$ and $U^{-} \cap V$. In order to prove the lemma, we just have to prove that $U^{+}$contains $V^{+}$and that $U^{-}$contains $V^{-}$. Since $U^{+}$is the union of the $c_{t}^{+}, U^{+} \cap V$ is actually the union of increasing and decreasing graphs.

We distinguish now two cases:

Case 1: The Anosov flow is not topologically equivalent to a suspension (see Fig. 6): We can apply the results of the Section 2. The set of rays is naturally identified with the leaf space $\mathcal{L}^{s}$. We can interpret the translation $\tau^{s}: \mathcal{L}^{s} \rightarrow \mathcal{L}^{s}$ as a permutation of rays. Nearly by definition, the fundamental regions are the open subsets bounded by $r$ and $\tau^{s}(r)$, where $r$ is a ray. Denote by $\rho$ the function from $U^{+}$into $\mathcal{L}^{s}$ which maps a point $s$ to the supremum in $\mathcal{L}^{s} \approx \mathbf{R}$ of the set of rays which intersect the complete line containing $s_{0}$ and $s$, i.e. the unique graph $c_{t}^{+}$containing $s$. This function is very similar to the functions $\alpha$ and $\beta$ defined in Section 2, and since $\alpha$ and $\beta$ are homeomorphisms, it is easy to deduce that $\rho$ is continuous, that the image of $\rho$ is the interval ]$r_{0}, \tau^{s}\left(r_{0}\right)\left[\right.$, and that two points in $U^{+}$have the same image by $\rho$ if and only if they belong to the same $c_{t}^{+}$.

Assume that $V^{+}$is not contained in $U^{+}$, i.e. that there is a ray $r_{1}$ in $V^{+}$which is not contained in $U^{+}$. The intersection between $r_{1}$ and $U^{+}$is a segment $] s_{1}^{-}, s_{1}^{+}$[. We consider here only the case $s_{1}^{+}<+\infty$; the other case $s_{1}^{-}>-\infty$ can be treated in a similar way. Let $t_{1}$ be the unique parameter for which $\rho\left(c_{t_{1}}^{+}\right)=r_{1}$. Observe that $t_{1}$ is positive. Then, for any positive $t$ smaller than $t_{1}, c_{t}^{+}$meets $r_{1}$. In other words, for $t$ in $\left[0, t_{1}\right]$, the $c_{t}^{+}$are graphs of continuous functions $\psi_{t}$ defined on $\left[r_{0}, r_{1}\left[\right.\right.$, taking value in $\mathbf{R}_{+}^{*}$, and such that:

$$
\begin{gathered}
\lim _{r \rightarrow r_{1}} \psi_{t}(r)<b \quad\left(0<t<t_{1}\right) \\
\lim _{r \rightarrow r_{1}} \psi_{t_{1}}(r)=+\infty .
\end{gathered}
$$


In the first equation, $b$ is the second coordinate of $s_{1}$ in $V \approx \mathbf{R}^{2}$. The second equation follows from the fact that $c_{t_{1}}^{+}$is an embedded path which does not intersect $r_{1}$.

Since the $c_{t}^{+}$are increasing graphs, the functions $\psi_{t}$ are increasing. Therefore, for $t$ less than $t_{1}$, the function $\psi_{t}$ takes value only in $[0, b]$. On the other hand, since the function $f^{+}$is continuous, $\psi_{t}(r)$ is continuous in the parameter $t$. We obtain a contradiction since $\psi_{t_{1}}$ admits arbitrarily large values. This contradiction shows that any ray contained in $V^{+}$belongs to $U^{+}$. Hence, $U^{+}$contains $V+$, and $U^{-}$contains $V^{-}$. This achieves the proof of the lemma in this case.

Case 2: the Anosov flow is topologically equivalent to a suspension: We will prove later that actually this case cannot occur (Proposition 5.1). But the impossibility of this case is still far from obvious.

In this case, every leaf of $\widetilde{\mathcal{F}}^{s}$ meets every leaf of $\widetilde{\mathcal{F}}^{u}$. It follows that the whole $P^{s}$ is the unique fundamental region. In other words, any complete line containing $s_{0}$ is the graph of a function $\psi_{t}: l_{0} \rightarrow r_{0}$. When $t$ is positive, $\psi_{t}$ is increasing, and when $t$ is negative, $\psi_{t}$ is decreasing. By continuity of $f^{+}$and $f^{-}$, the map $t \mapsto \psi_{t}(r)$ is continuous for every $r$. Let $g^{+}$(resp. $g^{-}$) be the limit of the functions $\psi_{t}$ when $t$ tends to $+\infty$ (resp. $-\infty$ ). The function $g^{+}: l_{0} \rightarrow r_{0} \cup\{+\infty\}$ is increasing, and $g^{-}: l_{0} \rightarrow r_{0} \cup\{-\infty\}$ is decreasing. Then, $U^{+}$(resp. $U^{-}$) is the open set in $V^{+}$ bounded by the graphs of $g^{+}$and $g^{-}$over the positive (resp. negative) part of $l_{0}$.

Assume for a moment that the ray $r_{0}$ is preserved by some element $\gamma$ of $\Gamma$. Then, there is a real number $T$ such that $r_{0}$ is fixed pointwise by $\varphi^{T} \circ \gamma$. There is a special parametrization of $P^{s}$ such that $s_{0}$ is of coordinates $(0,0)$, such that $r_{0}$ is the vertical line, and such that the horizontal line is the unique $\varphi^{T} \circ \gamma$-invariant complete line through $s$.

The action of $\varphi^{T} \circ \gamma$ on $P^{s}$ is of the form $(t, s) \mapsto(\kappa(t), s)$ where $\kappa$ is a contraction or a dilatation. But $\varphi^{T} \circ \gamma$ must preserve $U$, and therefore the boundary of it. Since $g^{+}$is increasing and $g^{-}$is decreasing, the only possibility is $g^{+}=+\infty$ and $g^{-}=-\infty$ (see Fig. 7).

In other words, when the ray $r_{0}$ through $s_{0}$ is invariant by some element of $\Gamma$, every point of $P^{s}$ belongs to a generalized line containing $s_{0}$.

We go back to the general case: what we did above shows that $U^{+} \cup U^{-} \cup r_{0}$ contains all the rays preserved by some element of $\Gamma$. The lemma follows since the union of these rays is dense

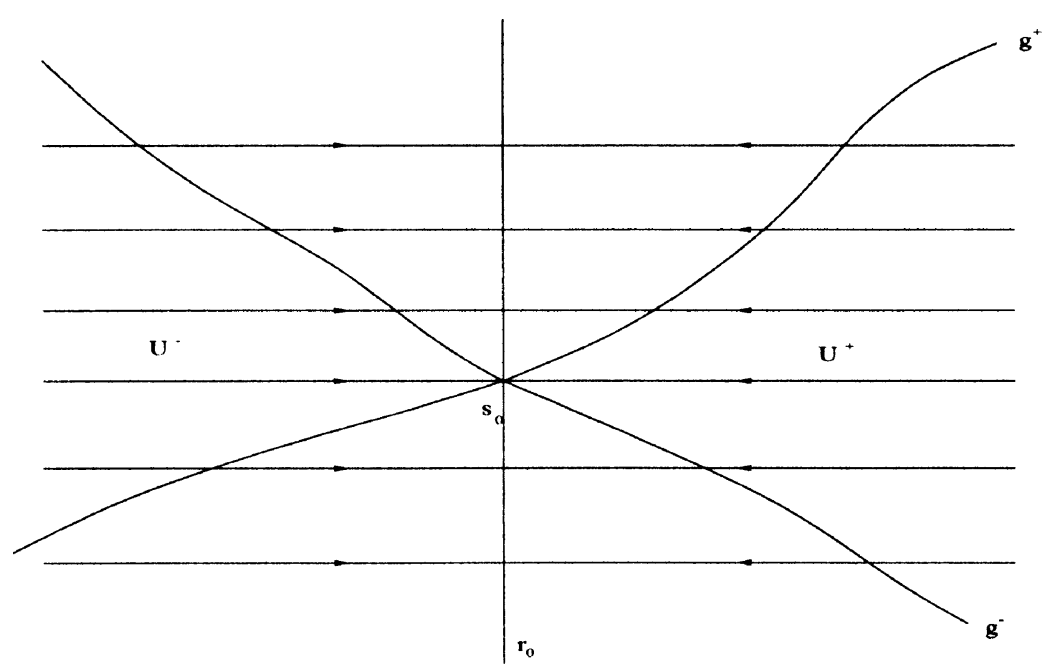

Fig. 7. Action of $\varphi^{T} \circ \gamma$. 
in $P^{s}$ (this last assertion follows from the density of periodic orbits of topologically transitive Anosov flows).

We proved all the statements necessary for the proof of Theorem B:

THEOREM 3.17. - The space $P^{s}$, equipped with the generalized lines defined above, is a lifted affine $\widetilde{\mathbf{R}}^{2}$-plane.

Proof. - We check the six axioms one by one:

(1) Axiom 1 is Proposition 3.13.

(2) Axiom 2 follows from Theorem 3.15 since the rays are the projections in $P^{s}$ of the leaves of $\widetilde{\mathcal{F}}^{s}$.

(3) The unicity part of Axiom 3 is Lemma 3.14. The existence part is Lemma 3.16.

(4) Axiom 4 is obvious since the parallels of a complete line are its $\varphi^{t}$-iterates.

(5) The TCP property is symmetric: we always privileged the stable foliation, but all what we did apply if we exchange the role of $\widetilde{\mathcal{F}}^{s}$ and $\widetilde{\mathcal{F}}^{u u}$. Let $P^{u}$ be the leaf space of $\widetilde{\mathcal{F}}^{u u}$ : according to our previous work, Axiom 3 is true in $P^{u}$. But $P^{u}$ can be considered as the dual of $P^{s}$, i.e. the set of complete lines of $P^{s}$. Axiom 5 is nothing but the dual version in $P^{s}$ of Axiom 3 in $P^{u}$.

(6) The topology that we have to consider on the set of generalized lines is obvious; and Axiom 6 is obvious also for this topology.

\section{Margulis measure and flag variety}

For any topologically transitive Anosov flow, Margulis ([24]) constructed a foliated measure supported on weak unstable leaves, depending continuously on the leaf, multiplied by the Anosov flow, and preserved by the holonomy along strong stable leaves. Hence, it induces a Borel measure $\nu$ on $P^{s}$ such that:

$-\nu$ is nonatomic, and any open subset is of positive $\nu$-measure,

$-\nu$ is preserved by the fundamental group $\Gamma$,

- there is a constant $\lambda$ such that, for any $t$, the measure is multiplied by $\lambda^{t}$ under the action of $\varphi^{t}$.

Moreover, the strong stable foliation is uniquely ergodic. ${ }^{1}$ It means that $\nu$ is the unique $\Gamma$ invariant Borel measure on $P^{s}$ up to constant factor.

DEFinition 4.1. - A $\Gamma$-invariant measure $\nu$ as above is called a Margulis measure.

We now define another important notion:

Definition 4.2. - Let $P$ be a lifted affine $\widetilde{\mathbf{R}}^{2}$-plane. The flag variety associated to $P$ is the set of pairs $(p, l)$ where $p$ is a point of $P$ and $l$ a complete line containing $p$.

The flag variety associated to any lifted affine $\widetilde{\mathbf{R}}^{2}$-plane is always homeomorphic to $\mathbf{R}^{3}$. Any collineation induces naturally a transformation on the flag variety. Consider a TCP Anosov flow $\Phi^{t}$. There is a natural map from the universal covering $\widetilde{M}$ onto the flag variety $F$ associated to $P^{s}$ : the image of a point $x$ of $\widetilde{M}$ is the pair $\left(\widetilde{\mathcal{F}}^{s s}(s), l\right)$, where $l$ is the projection in $P^{s}$ of $\widetilde{\mathcal{F}}^{u u}(x)$. Obviously, this map is a $\Gamma$-equivariant homeomorphism. Moreover, it maps the flow $\widetilde{\Phi}^{t}$ on the flow $\Psi^{t}$ induced by the collineations $\varphi^{t}$ of $P^{s}$.

\footnotetext{
${ }^{1}$ Unique ergodicity of strong foliations is proved in [6] when the Anosov flow is topologically mixing, and we will see that TCP Anosov flows are topologically mixing (Remark 5.4).

4 ${ }^{\text {e }}$ SÉRIE - TOME $34-2001-\mathrm{N}^{\circ} 6$
} 
It follows that the TCP Anosov flow $\Phi^{t}$ is topologically conjugate to the flow induced by $\Psi^{t}$ on the quotient of the flag variety $F$ by the collineation group $\Gamma$.

\section{Suspensions and the topological contact property}

\section{Proposition 5.1. - A TCP Anosov flow is not topologically equivalent to a suspension.}

Remark 5.2. - This proposition is well-known in the particular case of contact Anosov flow, but the proof is more subtle in the general TCP setting. Our proof is quite sophisticated and relies on all the previous results.

Remark 5.3. - According to a Theorem of V.V. Solodov, an Anosov flow on a 3-manifold is topologically equivalent to a suspension if and only if it has the splitting property, i.e. if every leaf of $\widetilde{\mathcal{F}}^{s}$ meets every leaf of $\widetilde{\mathcal{F}}^{u}$ ([32] or Theorem 2.7 of [2]). In the context of TCP Anosov flows, and according to lemma 3.16, the splitting property would mean that any two points in $P^{s}$ belong to some generalized line. Hence, an equivalent formulation of Proposition 5.1 is: the lifted affine $\widetilde{\mathbf{R}}^{2}$-plane associated to a TCP Anosov flow is never an affine $\mathbf{R}^{2}$-plane (see Remark 1.1).

Remark 5.4. - According to Remark 2.2, it follows that TCP Anosov flows are topologically mixing.

We will need the following definition:

Definition 5.5. - Let $I$ be a compact segment in a complete line of $P^{s}$. The radial triangle $T(I)$ of base $I$ is the union of all the $\varphi^{t}(I)$ for negative $t$.

Observe that different bases define different radial triangles. Let $\nu$ be a Margulis measure (see Definition 4.2).

LEMA 5.6. - Every radial triangle is of finite $\nu$-measure.

Proof. - Let $T$ be a radial triangle of base $I$. For any natural integer $i$, let $T_{i}$ be the union of the $\varphi^{t}(I)$ for $t$ in $[-i-1,-i]$. Every $T_{i}$ is compact. Hence, its $\nu$-measure $m_{i}$ is finite. The $\nu$-measure of $T$ is less than the sum of the $m_{i}$ 's. Since $\varphi^{t}$ multiplies $\nu$ by $\lambda^{t}$, the measure $m_{i}$ is less than $\lambda^{-i} m_{0}$. The lemma follows since $\lambda>1$.

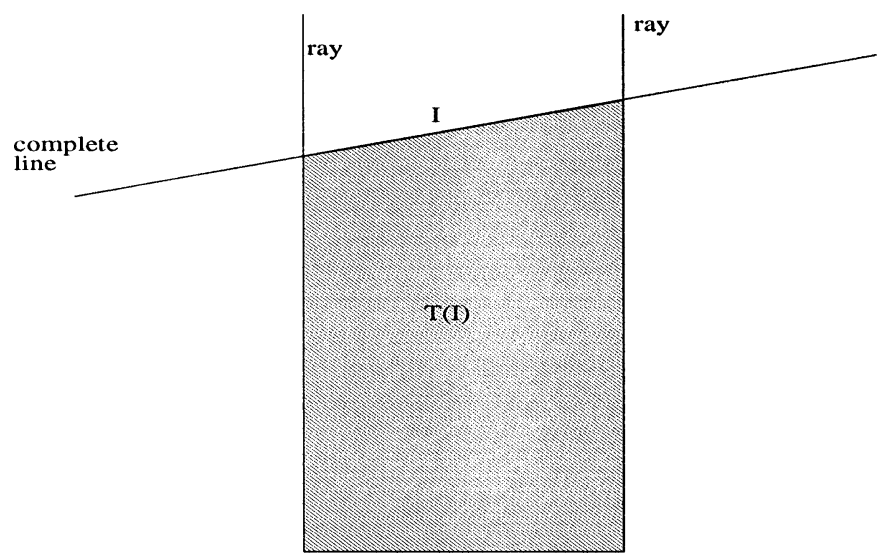

Fig. 8. A radial triangle. 
Proof of 5.1. - Assume a contrario that the TCP Anosov flow is topologically equivalent to the suspension of some Anosov diffeomorphism of the torus $T$. According to Remark 5.3, the whole $P^{s}$ is a fundamental region. According to Lemma 3.16, any pair of points in $P^{s}$ belongs to a generalized line. Since the flow is a suspension, the ambient manifold is a torus bundle over the circle. There is a cyclic covering $\widehat{M}$ of $M$ which is homeomorphic to $T \times \mathbf{R}$, and the lifting of $\Phi^{t}$ in $\widehat{M}$ is a flow $\widehat{\Phi}^{t}$ such that any orbit of $\widehat{\Phi}^{t}$ is a closed embedding of $\mathbf{R}$ which intersects every $T \times\{*\}$ in one and only one point. In particular, the orbit space of $\widehat{\Phi}^{t}$ is homeomorphic to $T$. The Galois group of the covering $\widehat{M} \rightarrow M$ is a normal subgroup $H$ of $\Gamma$ isomorphic to $\mathbf{Z}^{2}$. Since no leaf of $\mathcal{F}^{s s}$ is a fiber, the action of $\Gamma$ on $P^{s}$ is free. Actually, since $\widehat{\Phi}^{t}$ has no periodic orbit, $H$ acts freely on the set of rays of $P^{s}$. Select an element $a$ of $H$. Consider an element $m=(s, l)$ of the flag variety $F$ associated to $P^{s}$. Let $r$ be the ray through $s$ : the image $a(r)$ intersects the complete line $l$ at a unique point $s^{\prime}$. Then, $\left[s, s^{\prime}\right]$ is a compact segment of $l$. Denote by $T(m)$ the radial triangle of base $\left[s, s^{\prime}\right]$ (see Definition 5.5), and by $\mu(m)$ the $\nu$-measure of $T(m)$. Then, $\mu: F \rightarrow \mathbf{R}$ is a continuous function. Since any element of $H$ commutes with $a, \mu$ is $H$-invariant. Moreover, $\mu\left(\Psi^{t}(m)\right)=\lambda^{t} \mu(m)$, where $\Psi^{t}$ is the flow induced by $\varphi^{t}$ on $F$. Therefore, $\mu$ is not constant, and the set-locus $\mathcal{E}$ where $\mu$ equals one is a $H$-invariant closed subset of $F$ which meets every orbit of $\Psi^{t}$ at one and only one point. We have seen in the Section 5 that $\Phi^{t}$ is topologically conjugate to the flow induced by $\Psi^{t}$ on the quotient of $F$ by $\Gamma$. It follows that the quotient of $\mathcal{E}$ by $H$ is homeomorphic to the orbit space of $\widehat{\Phi}^{t}$, i.e. to $T$. Finally, $\mu$ is injective along every leaf of $\widetilde{\mathcal{F}}^{s s}$ since if $m=(s, l)$ and $m^{\prime}=\left(s, l^{\prime}\right)$ are two different points of the same strong stable leaf, one of the radial triangles $T(m)$ and $T\left(m^{\prime}\right)$ contains the other. It follows that $\mathcal{E}$ can be interpreted also as the leaf space of $\widetilde{\mathcal{F}}^{s s}$, i.e. $P^{s}$.

What we finally obtained is the following: the action of $H$ on $P^{s}$ is free and properly discontinuous; the quotient $Q$ of this action is homeomorphic to the torus T. The Borel measure $\nu$ induces a mesure $\bar{\nu}$ on the compact $Q$, and $\varphi^{t}$ induces a flow $\bar{\varphi}^{t}$ on $Q$. The measure $\bar{\nu}$ has a total mass which is finite and not null. But this measure is contracted by $\bar{\varphi}^{t}$ by the factor $\lambda^{t}$ : contradiction.

\section{Desarguian Anosov flows}

Definition 6.1. - A TCP Anosov flow is called Desarguian if the leaf space $P^{s}$ is affinely isomorphic to the universal covering of the usual punctured affine plane $\mathbf{R}^{2} \backslash\{0\}$.

As we have indicated in the introduction, an alternative definition of (generalized) geodesic flow of Riemanian surfaces with constant negative curvature is the following: consider a discrete uniform subgroup $\Gamma$ of $\widetilde{S L}(2, \mathbf{R})$, the universal covering of $S L(2, \mathbf{R})$. Let $\widetilde{\mathcal{R}}$ be the universal covering of $\mathbf{R}^{2} \backslash\{0\}$, viewed as a lifted affine $\widetilde{\mathbf{R}}^{2}$-plane. Observe that the affine action of $S L(2, \mathbf{R})$ on $\mathbf{R}^{2} \backslash\{0\}$ lifts as an action of $\widetilde{S L}(2, \mathbf{R})$ on $\widetilde{\mathcal{R}}$. The collineation group of $\widetilde{\mathcal{R}}$ contains another 1-parameter subgroup: the flow $\varphi_{0}^{t}$, lifting of the radial flow of $\mathbf{R}^{2} \backslash\{0\}$.

Let $F$ be the flag variety associated to $\widetilde{\mathcal{R}}$ (see Definition 4.2). It is equipped with an action of $\widetilde{S L}(2, \mathbf{R})$, which commutes with the flow $\Psi_{0}^{t}$ induced by $\varphi_{0}^{t}$. Let $M_{\Gamma}$ be the quotient of $F$ by $\Gamma$ : it is a 3-manifold (actually, a Seifert manifold). Let $\Phi_{\Gamma}^{t}$ be the flow induced by $\Psi_{0}^{t}$ on V. Then, $\Phi_{\Gamma}^{t}$ is an Anosov flow which is smoothly conjugate (up to finite covers) to the geodesic flow of a Riemannian surface with constant negative curvature. The strong stable leaves of $\Phi_{\Gamma}^{t}$ lifted in the universal covering are nothing but the fibers of the fibration of $F$ over $\widetilde{\mathcal{R}}$. It follows that $\Phi_{\Gamma}^{t}$ is Desarguian.

Now, we can modify the action of $\Gamma$ on $F$ : let $\rho: \Gamma \rightarrow \mathbf{R}$ be a morphism. We now define a new action of $\Gamma$ on $F$ : the element $\gamma$ of $\Gamma$ maps the element $x$ of $F$ on $\Psi_{0}^{\rho(\gamma)} \circ \gamma(x)$. Then, this new 
action of $\Gamma$ is still an action by collineations, and it commutes with $\Psi_{0}^{t}$. It happens that for some morphisms $\rho$, the new associated $\Gamma$-action on $F$ remains free and properly discontinuous. Then, the quotient is a 3 -manifold diffeomorphic to $M_{\Gamma}$, and $\Psi_{0}^{t}$ induces on this quotient an Anosov flow $\Phi_{\Gamma, \subset}^{t}$. The Anosov flows constructed in this way are the exotic Anosov flows defined in [15]: indeed, what we did here is just to reformulate the definition given in [15]. The fact that $\Phi_{\Gamma, \rho}^{t}$ is an Anosov flow is not completely obvious, we refer to [15] for the details. The liftings in $F$ of the leaves of the strong foliations of the exotic Anosov flows are still the fibers of the fibration $F \rightarrow \widetilde{\mathcal{R}}$. Therefore, the exotic Anosov flows are Desarguian. Conversely:

Proposition 6.2. - A TCP Anosov flow is Desarguian if and only if it is topologically conjugate to an exotic Anosov flow.

Proof. - Let $\Phi^{t}$ be a Desarguian TCP Anosov flow. Here, $\Gamma$ will denote the fundamental group of the ambient manifold of $\Phi^{t}$. The projection $\varphi^{t}$ of $\Phi^{t}$ in $P^{s}$ is a flow whose orbits are the rays of $\widetilde{\mathcal{R}}$, i.e. the liftings of the rays of $\mathbf{R}^{2} \backslash\{0\}$, and for every $t, \varphi^{t}$ is a collineation. It follows easily that, up to a constant factor on the parameter, $\varphi^{t}$ is the lifting $\varphi_{0}^{t}$ in $\widetilde{\mathcal{R}}$ of the usual radial flow of $\mathbf{R}^{2} \backslash\{0\}$. Let $F$ be the flag variety of $\widetilde{\mathcal{R}}$, and let $\Psi_{0}^{t}$ be the "radial flow" on $F$ induced by $\varphi_{0}^{t}$. Then, since $\varphi^{t}$ and $\varphi_{0}^{t}$ are equal, $\Phi^{t}$ is topologically conjugate to the flow $\Phi_{0}^{t}$, which is the flow induced by the radial flow $\Psi_{0}^{t}$ on the quotient of $F$ by $\Gamma$. In particular $\Phi_{0}^{t}$ is an Anosov flow, and the proposition will follow if we prove that $\Phi_{0}^{t}$ is topologically conjugate to an exotic Anosov flow.

Observe that $\Phi_{0}^{t}$ is smooth. Moreover, the lifting in $F$ of the strong stable leaves of $\Phi_{0}^{t}$ are the fibers of the projection of $F$ over $P^{s}$. Hence, the strong stable foliation of $\Phi_{0}^{t}$ is smooth. A similar argument shows that the strong unstable foliation is smooth also. By the main theorem of [15], $\Phi_{0}^{t}$ is smoothly conjugate to an exotic Anosov flow.

Remark 6.3. - The proof given here is short but very inelegant. The main difficulty in [15] consists in showing that if an Anosov flow has smooth strong foliations, then the leaf space of $\widetilde{\mathcal{F}}^{s s}$ is locally modelled on $\mathbf{R}^{2}$ so that the leaves of $\widetilde{\mathcal{F}}^{u u}$ project in this leaf space as straight lines (Proposition 3.7 of [15]). In our case, we know that immediately. The real difficulties here for the proof of Proposition 6.2 are:

- first, we have to show that the collineation group of e $F$ is $\widetilde{S L}(2, \mathbf{R}) \times \mathbf{R}$, where the factor $\mathbf{R}$ is the 1-parameter subgroup $\varphi_{0}^{t}$ : this is quite easy, but we shall not discuss it here.

- then, we have to show that any subgroup of $\widetilde{S L}(2, \mathbf{R}) \times \mathbf{R}$ which acts freely and properly discontinuously on $F$ is of the form $(\gamma, \rho(\gamma))$, where $\gamma$ describes a discrete uniform subgroup $\Gamma$ of $\widetilde{S L}(2, \mathbf{R})$, and where $\rho: \Gamma \rightarrow \mathbf{R}$ is a morphism. This is precisely the matter of Theorem 6.4 in [15].

Remark 6.4. - We can wonder if in Proposition 6.2 the topological conjugacy is actually smooth. Observe that it is true if $\Phi^{t}$ admits a smooth splitting. Moreover, it is known that geodesic flows of negatively curved closed surfaces are smoothly conjugate as soon as they are topologically conjugate. On the other hand, we do not know if any Anosov flow topologically conjugate to a geodesic flow is smoothly conjugate to it. When considering this question, we have to notice that its anologue in the context of suspensions is not true. Indeed, any Anosov diffeomorphism on the torus is topologically conjugate to a linear automorphism, but in general the conjugacy is not $C^{1}$.

Remark 6.5. - The main result of [15] can now be rewritten as follows: up to smooth conjugacies, the only Anosov flows in dimension 3 admitting smooth splittings are the Desarguian Anosov flows. 


\section{REFERENCES}

[1] Anosov D.V., Geodesic flows on closed Riemannian manifolds of negative curvature, Trudy Mat. Inst. Steklov. 90 (1967).

[2] BARвот T., Caractérisation des flots d'Anosov en dimension 3 par leurs feuilletages faibles, Ergodic Theory Dynam. Systems 15 (1995) 247-270.

[3] Barbot T., Flots d'Anosov sur les variétés graphées au sens de Waldhausen, Ann. Inst. Fourier (Grenoble) 46 (1996) 1451-1517.

[4] Ваввот T., Generalizations of the Bonatti-Langevin example of Anosov flow and their classification up to topological equivalence, Comm. Anal. Geom. 6 (1998) 749-798.

[5] Bonatti C., Langevin R., Un exemple de flot d'Anosov transitif transverse à un tore et non conjugué à une suspension, Ergodic Theory Dynam. Systems 14 (1994) 633-643.

[6] Bowen R., MARcus B., Unique ergodicity for horocycle foliations, Israel J. Math. 26 (1) (1977) 43-67.

[7] Buekenhout F., Handbook of Incidence Geometry, North-Holland, Amsterdam, 1995, Edited by F. Buekenhout, 1420 pp.

[8] Fenley S.R., Anosov flows in 3-manifolds, Ann. of Math. (2) 139 (1) (1994) 79-115.

[9] Fenley S.R., The structure of branching in Anosov flows of 3-manifolds, Comment. Math. Helv. 73 (2) (1998) 259-297.

[10] FOULON P., private communication.

[11] Franks J., Anosov diffeomorphisms, in: Global Analysis (Berkeley, Calif., 1968), Proc. Sympos. Pure Math., Vol. XIV, American Mathematical Society, Providence, RI, 1970, pp. 61-93.

[12] Franks J., Williams B., Anomalous Anosov flows, in: Lectures Notes in Math., Vol. 819, 1980, pp. 158-174.

[13] FRIED D., Transitive Anosov flows and pseudo-Anosov maps, Topology 22 (1983) 299-303.

[14] Ghys E., Flots d'Anosov sur les 3-variétés fibrées en cercles, Ergodic Theory Dynam. Systems 4 (1) (1984) 67-80.

[15] Ghys E., Flots d'Anosov dont les feuilletages stables sont différentiables, Ann. Sci. École Norm. Sup. (4) 20 (2) (1987) 251-270.

[16] Ghys E., Déformations de flots d'Anosov et de groupes fuchsiens, Ann. Inst. Fourier (Grenoble) 42 (1-2) (1992) 209-247.

[17] GHYs E., Rigidité différentiable des groupes fuchsiens, Inst. Hautes Études Sci. Publ. Math. 78 (1993) 163-185.

[18] Goodman S., Dehn surgery on Anosov flows, in: Lectures Notes in Math., Vol. 1007, 1983, pp. 300307.

[19] Handel M., Thurston W., Anosov flows on new three manifolds, Invent. Math. 59 (1980) 95-103.

[20] Hasselblatt B., KatoK A., Introduction to the Modern Theory of Dynamical Systems (With a supplementary chapter by A. Katok and L. Mendoza), Encyclopedia of Mathematics and its Applications, Vol. 54, Cambridge University Press, Cambridge, 1995.

[21] Hasselblatt B., Wilkinson A., Prevalence of non-Lipschitz Anosov foliations, Ergodic Theory Dynam. Systems 19 (1998) 643-656.

[22] Hirsch M.W., Pugh C., Stable manifolds and hyperbolic sets, in: Global Analysis (Berkeley, Calif., 1968), Proc. Sympos. Pure Math., Vol. XIV, American Mathematical Society, Providence, RI, 1970, pp. 133-163.

[23] HURDER S., KATOK A., Differentiability, rigidity and Godbillon-Vey classes for Anosov flows, Inst. Hautes Études Sci. Publ. Math. 72 (1990) 5-61.

[24] Margulis G.A., Certain measures that are connected with U-flows on compact manifolds, Functional Anal. Appl. 4 (1970) 55-67.

[25] Newhouse S.E., On codimension one Anosov diffeomorphisms, Amer. J. Math. 92 (1970) 761-770.

[26] Palmeira C.F.B., Open manifolds foliated by planes, Ann. Math. 107 (1978) 109-131.

[27] Plante J.F., Anosov flows, Amer. J. Math. 94 (1972) 729-754.

[28] Plante J.F., Anosov flows, transversely affine foliations, and a conjecture of Verjovsky, J. London Math. Soc. (2) 23 (2) (1981) 359-362. 
[29] Plante J.F., Thurston W., Anosov flows and the fundamental group, Topology 11 (1972) 147-150. [30] Salzmann H., Betten D., Grundhöfer T., Hähl H., LöWen R., Stroppel M., Compact Projective Planes, De Gruyter Expositions in Mathematics, Vol. 21, Walter de Gruyter, Berlin, 1995.

[31] Simić S., Codimension one Anosov flows and a conjecture of Verjovsky, Ergodic Theory Dynam. Systems 17 (1997) 1221-1231.

[32] Solodov V.V., The universal cover of Anosov flows, preprint, 1992.

[33] Thurston W., Three-manifolds, foliations and circles, I, preprint, 1997, math.gt/9712268.

[34] Verjovsky A., Codimension one Anosov flows, Bol. Soc. Mexicana (2) 19 (2) (1974) 49-77.

(Manuscript received April 28, 2000; accepted, after revision, October 19, 2000.)

\author{
Thierry BARBOT \\ ENS Lyon, UMPA, \\ UMR 5669 du C.N.R.S., \\ 46, allée d'Italie, \\ 69364 Lyon, France \\ E-mail: barbot@umpa.ens-lyon.fr
}

